\section{CPS-067 EFFECTIVENESS AND SAFETY OF THE EARLY SWITCH FROM INTRAVENOUS TO ORAL ANTIBIOTICS TREATMENT IN THE PNEUMOLOGY WARD}

${ }^{1} \mathrm{D}$ Echeverria-Esnal, ${ }^{1} \mathrm{~J}$ Martinez-Casanova, ${ }^{2} \mathrm{M}$ Dominguez-Alvarez, ${ }^{2} \mathrm{C}$ Estirado, ${ }^{2} \mathrm{MP}$ Ausin, ${ }^{2}$ E Balcells, ${ }^{2} \mathrm{C}$ Martin-Ontiyuelo, ${ }^{1} \mathrm{P}$ Acin, ${ }^{1} \mathrm{E}$ Navarrete Rouco*${ }^{2}{ }^{2} \mathrm{~J} \mathrm{Gea},{ }^{1} \mathrm{~S}$ Grau. ${ }^{1} \mathrm{Hospital}$ Del Mar, Pharmacy, Barcelona, Spain; ${ }^{2}$ Hospital Del Mar, Respiratory, Barcelona, Spain

10.1136/ejhpharm-2019-eahpconf.216

Background The intravenous administration of antibiotics remains the route of choice at patient admission. Although the early-oral switch once the clinical stability has been achieved, has demonstrated to be effective and safe in other settings, its implementation in the pneumology ward has not been studied.

Purpose To assess the effectiveness and safety of an early-oral antimicrobial switch protocol in the pulmonology ward.

Material and methods Quasi-experimental study performed in a 400-bed tertiary hospital. The protocol was implemented in March 2018 and therefore two groups were identified: intervention group (March to August 2018) and control group (March to August 2017). All patients admitted to the pneumology ward were treated with intravenous antibiotics that, in turn, were available orally: therefore, amoxicillin/clavulanate, fluoroquinolones, trimethoprim-sulfamethoxazole, clindamycin and azithromycin were included.

Results A total of 200 patients were included. Main clinical outcomes are summarised in table 1.

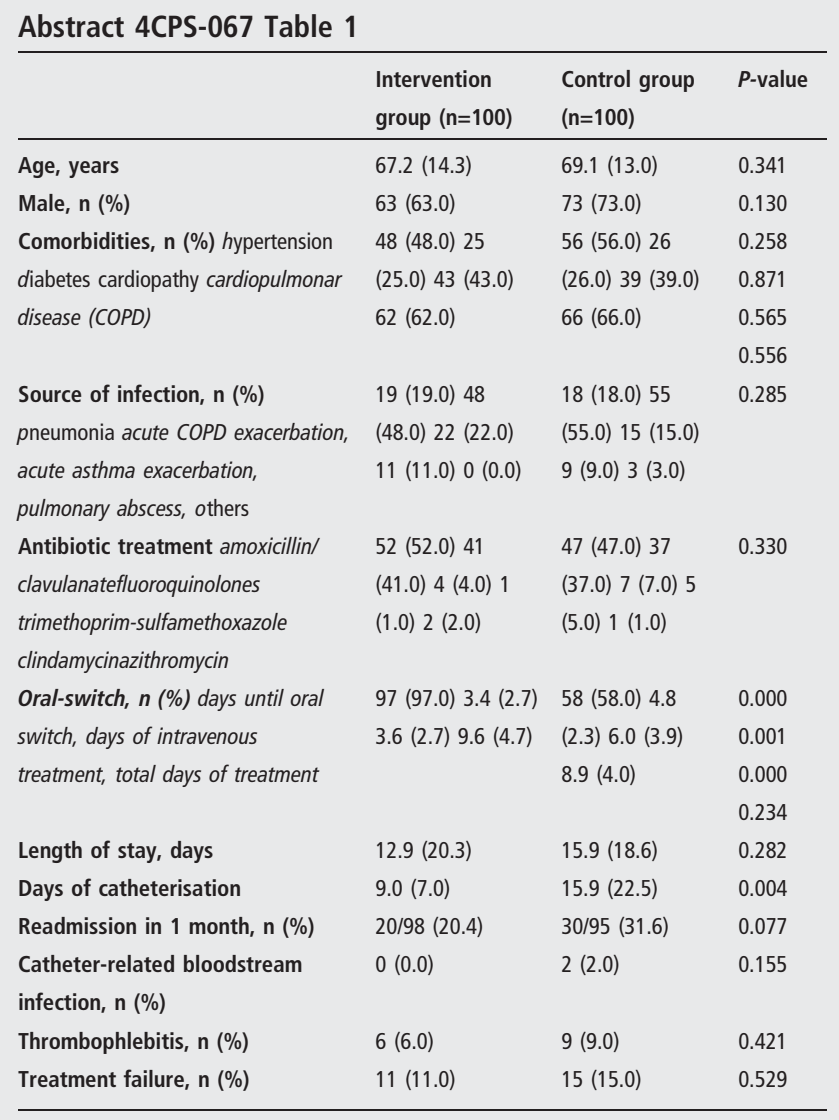

Conclusion The implementation of an early-oral antimicrobial switch protocol in the pneumology ward is effective and safe.
The early-oral antibiotic switch could decrease the days of catheterisation and the potential related adverse outcomes, with a shortening in the length of stay.

\section{REFERENCES AND/OR ACKNOWLEDGEMENTS}

None.

No conflict of interest.

\section{CPS-068 IMPACT OF THE PHARMACEUTICAL RECOMMENDATIONS FOR THE IMPROVEMENT IN ANTIBIOTICS PRESCRIPTION}

C Notario Dongil* ${ }^{*}$ A Marcos de la Torre, MT Gomez Lluch, M Malañon Pardo, J Martinez Mayordomo, JL Sanchez Serrano, P Araque Arroyo. Hospital General la Mancha Centro, Hospital Pharmacy Service, Alcazar de San Juan, Spain

\subsection{6/ejhpharm-2019-eahpconf.217}

Background Antibiotic prescription has been increased over the past years. A misuse of them has led to an increasing antibiotic-resistant bacteria and side effects in patients. Thus, the goal of pharmaceutical recommendations is to avoid these important issues.

Purpose To analyse the pharmaceutical interventions in antibiotics prescription performed in a third-level hospital and the degree of acceptance per service.

Material and methods A retrospective, observational and descriptive study of the pharmaceutical interventions in antibiotic prescription in our hospital over a 12 month period has been done. Types of interventions were collected from the antibiotic prescription of patients.

Pharmaceutical interventions registered were: incorrect dosage (ID), excessive duration (ED), incorrect dosage regime (IDR), pharmaceutical substitution (PS), de-escalation (DE) and other interventions (OI). We also analysed the degree of acceptance of these recommendations per services.

Data were collected from an electronic prescription program (Farmatools v. 2.6)

Results During the study period, 312 antibiotic interventions have been registered and analysed.

Prescribing services were: internal medicine 56\%, emergency department 11\%, surgery 9\%, urology 5\%, oncology and haematology $6 \%$, digestive $4 \%$, pneumology $3 \%$, traumatology $3 \%$, intensive care unit $2 \%$ andotorhinolaryngology $1 \%$.

Prescribed antibiotic families were: 154 beta-lactam antibiotics (49\%), 106 fluoroquinolones (34\%), 16 other antibacterials (5\%), 14 aminoglycosides (5\%), 13 glycopeptides (4\%), seven macrolides (2\%) and two sulfonamides (1\%).

Pharmaceutical interventions were: ID 40\%, ED 26\%, IDR $22 \%$, OI $5 \%$, PS $4 \%$ and DE $3 \%$.

The degree of acceptance of these recommendations was $42 \%$. We could not evaluate if $58 \%$ interventions were accepted. Acceptance per service was: internal medicine 49\%, surgery $15 \%$, oncology and haematology $8 \%$, urology $7 \%$, digestive $6 \%$, emergency department $5 \%$, traumatology $5 \%$, intensive care unit $3 \%$ and pneumology $2 \%$.

Conclusion The antibiotic family with the highest number of interventions was beta-lactam antibiotics. The most frequent intervention registered was ID. Internal medicine service accepted the highest number of interventions.

Data shows that the pharmaceutical role is important in achieving the correct antibiotic prescription. The objective of 
these recommendations will help to avoid antibiotic-resistance and side effects in patients.

\section{REFERENCES AND/OR ACKNOWLEDGEMENTS}

https://ejhp.bmj.com/content/25/1/1

No conflict of interest.

\section{CPS-069 IMPACT OF IMPLEMENTING A GLOBAL COLLABORATIVE PHYSICIAN-PHARMACIST STRATEGY ON PROPHYLACTIC ANTIBIOTIC PRACTICES IN A UNIVERSITY HOSPITAL CENTRE} Charleroi, Pharmacy, 6042 Lodelinsart, Belgium; ${ }^{2}$ Marie Curie Civil Hospital - Chu Charleroi, Infectious Diseases, 6042 Lodelinsart, Belgium; ${ }^{3}$ University of Mons, Mathematics, 7000 Mons, Belgium; ${ }^{4}$ Marie Curie Civil Hospital - Chu Charleroi, Cardiothoracic Surgery, 6042 Lodelinsart, Belgium

10.1136/ejhpharm-2019-eahpconf.218

Background Among different approaches to prevent surgical site infections, antibiotic prophylaxis is substantially important. According to previous published papers, surgical antibiotic prophylaxis (SAP) practices could be optimised by the implementation of isolated strategies.

Purpose

- To identify risk factors associated with non-compliance towards prophylactic antibiotic guidelines.

- To test the impact of a combined intervention strategy on compliance towards prophylactic antibiotic guidelines.

Material and methods A quasi-experimental study with a pretest-post-test evaluation was carried out on five types of intervention: hip prosthesis, coronary artery bypass grafting, colorectal surgery, transurethral resection of the prostate and endoscopic retrograde cholangiopancreatography. Compliance with guidelines was evaluated in 11 criteria within the pre-test and intervention groups.

- In order to identify risk factors associated with noncompliance, a retrospective observational transversal study was carried out in the pre-test group using a multivariate statistical analysis (Wald test). Odds ratios for the relationships between each independent variable and the outcome variable were then determined.

- We tested a combined intervention strategy that included: the pre-operative delivery of nominative kits containing the antibiotics with a recommendation paper adapted to patient factors; a pharmacist participating in antibiotic stewardship team for compilation of guidelines and their distribution for implementation; audits; feedback; educational seminar and outreach visits; and the development of an internal computer-based decision tool. For comparison between the two groups (pre-test and intervention groups), data were analysed using $\chi^{2}$ and t tests for, respectively, categorical and continuous data.

Results The pre-test group (11 January 2016 - 22 April 2016) and the intervention group (9 January 2017 - 21 April 2017) included, respectively, 130 and 118 interventions.

- The multivariate statistical analysis showed, as in previous studies, that true penicillin allergy, certain types of surgery and some practitioners were associated with non-compliance within the pre-test group.
- Compared with the pre-test group, the compliance was significantly increased in the test group for all 11 criteria $(\mathrm{P}<0.05)$ and in terms of global compliance $(42.4 \%$ vs $16.9 \% ; \mathrm{P}<0.001)$. This positive impact revealed a culture change, an interest and an awareness observed within the practitioner's teams.

Conclusion This study shows that optimisation of SAP practices is achievable within a proactive multidisciplinary approach.

\section{REFERENCE AND/OR ACKNOWLEDGEMENTS}

Wiffen P. Eur J Hosp Pharm 2018;25:1.

https://ejhp.bmj.com/content/25/1/1

No conflict of interest.

\section{CPS-070 DOES ANTIBIOTIC CONSUMPTION PREDICT THE INCIDENCE DENSITY OF HEALTHCARE-ASSOCIATED INFECTIONS?}

${ }^{1} \mathrm{~A}$ Peric*, ${ }^{2} \mathrm{~S}$ Vezmar Kovačevič, ${ }^{3} \mathrm{~B}$ Milenkovič, ${ }^{4} \mathrm{~V}$ Šuljagič. 'Military Medical Academy Faculty of Medicine, Sector of Pharmacy, Belgrade, Serbia; ${ }^{2}$ Faculty of Pharmacy- University of Belgrade, Department of Pharmacokinetics and Clinical Pharmacy, Belgrade, Serbia; ${ }^{3}$ Military Medical Academy, Sector of Pharmacy, Belgrade, Serbia; ${ }^{4}$ Military Medical Academy-Medical Faculty, Department of Healthcare-Related Infection Control, Belgrade, Serbia

10.1136/ejhpharm-2019-eahpconf.219

Background The decrease in healthcare associated infections (HAI) in intensive care units (ICUs), related to surgical-site infections (SSIs) and Clostridium difficile infections (CDIs), as well as antibiotic consumption, are the main goals in the hospital setting. Purpose The aim of this study was to evaluate the antibiotic consumption, and to relate it with HAI incidence density (ID) and incidence rate (IR).

Material and methods The study was conducted from 2011 to 2016 in a tertiary hospital. Through regular hospital surveillance, we identified all patients with a new HAI. Data on the use of antibacterials for systemic use were expressed as defined daily dose per 100 bed days (DDD/100 BD).

Results The highest ID of HAIs was observed in patients in surgical ICUs (25.5-47.2/1000 patient days), while the IR of SSI was $3.7 \%$. Moreover, the highest ID of CDI in medical patients was 6.2 , while in surgical patients it was 4.3 per 10000 patient days, while, at the same time, the antibiotic consumption was the lowest (31.2 DDD/100 BD). The most frequently used antibiotics, on average, were cephalosporins, aminoglycosides and carbapenems (16.0 $2.3,4.8 \pm 0.7,4.3$ $\pm 0.7 \mathrm{DDD} / 100 \mathrm{BD}$, respectively). The decrease in use of glycopeptides and fluorochinolones was predictive of higher ID of medical CDIs $(\mathrm{p}<0.05)$.

Conclusion The most frequently used antibiotics were not associated with HAIs. However, the decrease in use of glycopeptides and flurochinolones was associated with higher ID of CDIs. Simply decreasing the consumption of antibiotics with high risk for HAIs may not be sufficient.

\section{REFERENCES AND/OR ACKNOWLEDGEMENTS}

1. Berríos-Torres SI, Umscheid CA, Bratzler DW, et al. Centers for disease control and prevention guideline for the prevention of surgical site infection, 2017. JAMA Surg 2017;152:784-91.

2. World Health Organisation (WHO) Collaborating Centre for Drug Statistics and Methodology. Guidelines for the ATC classification and DDD assignment, 2017. Oslo, 2016.

3. Davey $P$, Brown $E$, Charani $E$, et al. Interventions to improve antibiotic prescribing practices for hospital inpatients (Review). Coch Data Syst Rev 2017; 2:CD003543. 